\title{
Agronomic characterization and chemical composition of sorghum hybrids for silage making in rainfed conditions
}

\section{Caracterização agronômica e composição química entre híbridos de sorgo para silagem em condições de sequeiro}

\author{
Alex Lopes da Silva ${ }^{1 *}$; Ricardo Loiola Edvan²; Leilson Rocha Bezerra ${ }^{3}$; Marcos \\ Jácome de Araújo ${ }^{2}$; José Hamilton da Costa Filho ; Jacira Neves da Costa Torreão; \\ Diego Sousa Amorim ${ }^{6}$; Romilda Rodrigues do Nascimento ${ }^{7}$
}

\section{Highlights:}

There are large morphological variations between sorghum hybrids.

Forage production among sorghum hybrids varies up to $61.76 \mathrm{t} \mathrm{ha}^{-1}$.

The chemical composition of sorghum is influenced by its morphology.

\begin{abstract}
The sorghum crop stands out in animal feeding, as this feedstuff can be preserved still fresh in the form of silage. This study was conducted to determine the agronomic characterization and chemical composition of sorghum hybrids in rainfed conditions aiming at silage production. Twenty-five sorghum hybrids were used in a randomized-block experimental design with three replicates. The following variables were evaluated: number of tillers; plant height; percentages of leaf, stem, panicle, grain and dead material; leaf:stem ratio; live:dead material ratio; total fresh matter yield; total dry matter yield; yields of leaf, stem, panicle, grain and dead material (in t ha ${ }^{-1} \mathrm{DM}$ ); and chemical composition. Data were analyzed by the Scott-Knott procedure at the 5\% significance level. The greatest observed heights were 214.53, 229.00 and $240.07 \mathrm{~cm}$, found in hybrids SF15, SF25 and PROG134IPA, respectively. There was an effect for morphological characterization $(\mathrm{P}<0.001)$, with the best means observed in hybrids 9929030 and 9929026. The highest total fresh matter yields were found in SF15, SF11, 12F042150 (93.95, 82.61 and $79.27 \mathrm{t} \mathrm{ha}^{-1}$, respectively). Adequate dry matter contents for ensiling were observed in hybrids 9929030, 12F042224, 12F042150, FEPAGRO18, FEPAGRO19, FEPAGRO11, SF15, SF25, 1141572, 1141570 and BRS Ponta Negra. In terms of crude protein content, hybrids 9929030, FEPAGRO18, FEPAGRO11, 9929026, 947072, 947252, 12F042226 and BRS Ponta Negra stood out with values between 6.55 and 8.64\%. Hybrids 9929030, 12F042224, 12F042150, FEPAGRO19, FEPAGRO11, 1141572 and 1141570 exhibited potential for silage production.
\end{abstract}

Key words: Crude protein. Genotypes. Morphology. Sorghum bicolor.

\footnotetext{
${ }^{1}$ Discente do Curso de Doutorado do Programa de Pós-Graduação Integrado em Zootecnia, PDIZ, Universidade Federal da Paraíba, UFPB, Departamento de Zootecnia, Areia, PB, Brasil. E-mail: alex.lopes77@hotmail.com

2 Profs., Universidade Federal do Piauí, UFPI, Departamento de Zootecnia, Bom Jesus, PI, Brasil. E-mail: edvan@ufpi.edu.br; jacome@ufpi.edu.br

3 Prof., Universidade Federal de Campina Grande, UFCG, Centro de Saúde e Tecnologia Rural, Patos, PB, Brasil. E-mail: leilson@ ufpi.edu.br

4 Prof., Universidade Federal do Rio Grande do Norte, UFRN, Departamento Engenharia Agronômica, Macaíba, RN, Brasil. E-mail: hamilton_costa@yahoo.com.br

5 Profa, Ensino Básico, Técnico e Tecnológico do Colégio Técnico de Bom Jesus, CTBJ, Bom Jesus, PI, Brasil. E-mail: jacira. torreao@ufpi.edu.br

6 Discente do Curso de Doutorado do Programa de Pós-Graduação Integrado em Zootecnia, PDIZ, Universidade Federal do Ceará, UFC, Departamento de Zootecnia, Fortaleza, CE, Brasil. E-mail: diego.zootecnista@hotmail.com

7 Discente do Curso de Doutorado do Programa de Pós-Graduação em Ciência Animal, PPGCA, UFPI, Teresina, PI, Brasil. E-mail: romilda0155@hotmail.com

* Author for correspondence
} 
A produção do sorgo se destaca na alimentação animal por permitir a sua utilização para conservação verde como silagem. Objetivou-se determinar a caracterização agronômica e composição química entre híbridos de sorgo em condições de sequeiro visando a produção de silagem. Foram utilizados 25 híbridos de sorgo em delineamento em blocos casualizados com três repetições. Avaliou-se número de perfilhos, altura planta, porcentagens de folha, colmo, panícula, grão e material morto, relações de folha:colmo e material vivo:material morto, produtividade total de massa verde e seca e produtividade de folha, colmo, panícula, grãos e material morto em $\mathrm{t} \mathrm{ha}{ }^{-1} \mathrm{MS}$ e composição química. Os dados foram analisados pelo procedimento Scott-Knott ao nível 5\% de significância. Maiores alturas observadas foram de 214,53, 229,00 e 240,07 cm para os híbridos SF15, SF25 e PROG134IPA, respectivamente. Houve efeito na caracterização morfológica $(\mathrm{P}<0,001)$ em que as melhores médias foram observadas nos $9929030 \mathrm{e}$ 9929026. Maior produtividade total de massa verde foi constatada em SF15, SF11, 12F042150 com 93,95, 82,61 e 79,27 t ha-1, respectivamente. Já para teor de matéria seca adequado para ensilagem, foi obtido nos 9929030, 12F042224, 12F042150, FEPAGRO18, FEPAGRO19, FEPAGRO11, SF15, SF25, 1141572, 1141570 e BRS Ponta Negra. Com relação à proteína bruta, destacaram-se 9929030, FEPAGRO18, FEPAGRO11, 9929026, 947072, 947252, 12F042226 e BRS Ponta Negra com teores entre 6,55 a 8,64\%. Os híbridos 9929030, 12F042224, 12F042150, FEPAGRO19, FEPAGRO11, 1141572 e 1141570 apresentam potencial para produção de silagem.

Palavras-chave: Genótipos. Morfologia. Proteína bruta. Sorghum bicolor.

\section{Introduction}

Tropical climate regions are characterized by the occurrence of irregular rainfall events. Such conditions make it difficult to manage animals grazing on pasture, since plant growth is limited during the period of low precipitation. This scenario warrants the use of intensified systems, which then increases the demand for silages of high biological value coupled with high yield capacity, high nutritional value and efficient nutrient production per area (Cândido et al., 2002).

In rainfed conditions, approximately $80 \%$ of the herbage is produced during the rainy period. This herbage can be preserved in the form of silage to be used in the feeding of ruminants. When pasture grasses are not used in this practice, plants such as corn and sorghum are those most commonly cultivated to be ensiled. The use of sorghum plants adapted to the local environmental conditions as well as adequate crop management are important factors to maximize crop yield, since sorghum tolerates water deficits, which constitutes a great advantage when grown in tropical regions.
The sorghum crop stands out in animal feeding because it can be preserved fresh as silage or grazed directly. Sorghum can be planted for grain production, but some hybrids and cultivars may also be dual-purpose. One of the advantages of dual-purpose sorghum is that it can be used for silage making or grain production. Additionally, the sorghum plant can be harvested twice, i.e., it regrows after the first harvest, under favorable climatic conditions.

When developing sorghum hybrids, breeders aim at high yield performance. This would be manifested through total fresh and dry matter yields and growth traits plant height and survival, mainly, which are of extreme importance in the evaluation of sorghum (Cunha \& Lima, 2010). Likewise, morphological components such as leaf, stem, panicle and grains, as well as chemical composition, are necessary variables in the evaluation of different hybrids for silage making. The water content of a forage plant must also be taken into account, with values between 65 and $70 \%$ being considered adequate for efficient storage of the ensiled material (Possenti et al., 2005). 
The Sorghum bicolor species comprises a wide array of chemical compositions due to the many existing hybrids and their different productive abilities, i.e., grain, forage or dual-purpose (Avelino et al., 2011). Moreover, there is a need for hybrids adapted to the conditions encountered in each region. On this basis, the present study proposes to determine the agronomic characterization and chemical composition of sorghum hybrids in rainfed conditions aiming at silage production.

\section{Materials and Methods}

\section{Location}

The experiment was conducted from January to August 2014 at the Federal University of Piauí, Professora Cinobelina Elvas campus (CPCE), located in Bom Jesus - PI, Brazil (09 04' 26" S; $44^{\circ}$ $21^{\prime} 32$ " W, $277 \mathrm{~m}$ above sea level). The climate of the region is an Aw type - tropical with dry winters (Köppen \& Geiger, 1928). Precipitation and mean temperature data recorded during the sorghum hybrid cultivation period (January 10 to May 24, 2014) were obtained from the National Meteorology Institute [INMET] (2014) and are shown in Figure 1.

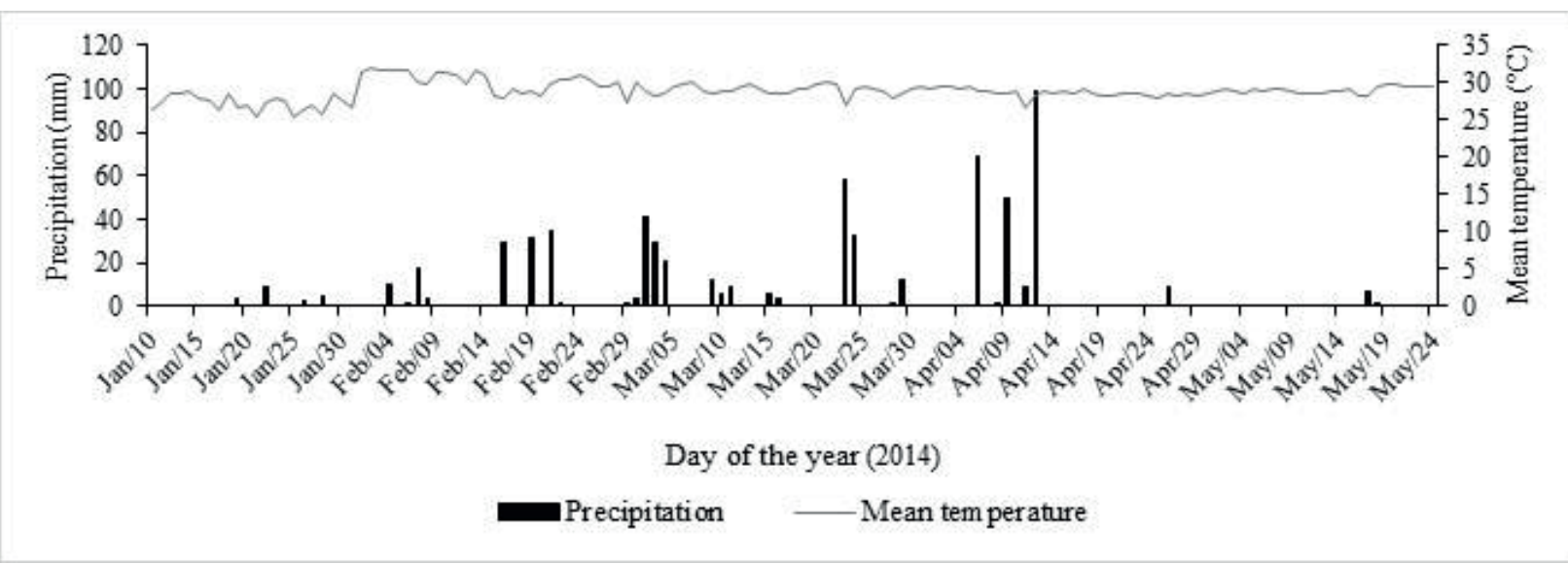

Source: Data from INMET (2014). Station: 82975 - Bom Jesus, Piauí, Brazil.

Figure 1. Precipitation $(\mathrm{mm})$ and mean temperature $\left({ }^{\circ} \mathrm{C}\right)$ data during the sorghum hybrid cultivation period.

\section{Experimental design and tested hybrids}

The experiment was laid out in a randomizedblock design with 25 sorghum hybrids and three replicates. The following hybrids were tested: 9929036, 9929030, 12F042224, 12F042150, FEPAGRO18, FEPAGRO19, FEPAGRO11, 9929012, 9929026, 947216, 947030, 947254, 947072, 947252, SF15, SF11, SF25, PROG134IPA, 12F042140, 12F042066, 12F042226, 12F042422, 12F042226, BRS506 and BRS Ponta Negra, all of which were provided by the Embrapa Maize and Sorghum corporation.

\section{Planting and fertilization}

The $620-\mathrm{m}^{2}$ area was divided into 75 plots measuring $4 \mathrm{~m}^{2}(2 \times 2 \mathrm{~m})$ each, with rows spaced $0.50 \mathrm{~m}$ apart, in a total of five rows per plot and 2.00 $\mathrm{m}$ between blocks. The seeds of all hybrids were distributed into furrows approximately $3 \mathrm{~cm}$ deep, at the rate of 14 seeds per linear meter, aiming at a final population of 140.000 plants ha ${ }^{-1}$ at harvest.

Soil from the planting area was sampled by following methods suggested by the Agronomic Institute of Paraná [IAPAR] (1996). A composite sample was extracted from sub-samples collected 
at random using a Dutch-type auger at a depth of 0 to $20 \mathrm{~cm}$. Chemical analyses (Table 1) were performed following the methodology of F. S. Silva (2011). On planting fertilization, $40 \mathrm{~kg} \mathrm{P} \mathrm{ha}^{-1}$ (single superphosphate) and $60 \mathrm{~kg} \mathrm{~K} \mathrm{ha-1} \mathrm{(potassium}$ chloride) were applied, and 40 days after planting, $70 \mathrm{~kg} \mathrm{~N} \mathrm{ha}^{-1}$ (urea) were applied, in accordance with the recommendations of Sousa and Lobato (2004).

Table 1

Chemical analysis of the soil where the 25 sorghum hybrids were planted

\begin{tabular}{|c|c|c|c|c|c|c|c|c|c|c|c|c|}
\hline $\mathrm{pH}$ & $\mathrm{P}$ & $\mathrm{K}$ & $\mathrm{Na}$ & $\mathrm{Ca}$ & $\mathrm{Mg}$ & $\mathrm{Al}$ & $\mathrm{H}+\mathrm{Al}$ & SB & $\mathrm{t}$ & $\mathrm{T}$ & $\mathrm{BS}$ & ASI \\
\hline $\mathrm{H}_{2} \mathrm{O}$ & \multicolumn{4}{|c|}{-----mg dm ${ }^{-3}-----$} & \multicolumn{6}{|c|}{ 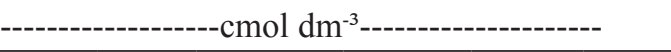 } & \multicolumn{2}{|c|}{-------\%------ } \\
\hline 5.78 & 29.6 & 84 & - & 2.8 & 1.2 & 0.1 & 3.3 & 4.32 & 4.32 & 7.52 & 56.09 & 2.32 \\
\hline
\end{tabular}

$\mathrm{pH}$ in water (ratio) - 1:2.5; P - K - Na - Mehlich 1 extraction; $\mathrm{Ca}-\mathrm{Mg}-\mathrm{Al}$ - KCl 1 mol L-1 extraction; H+Al - SMP extraction; SB - sum of exchangeable bases; CEC ( $\mathrm{t}$ ) - effective cation exchange capacity; CEC (T) - effective cation exchange capacity at pH 7.0; BS: base saturation index; ASI: aluminum saturation index.

\section{Agronomic traits and evaluation procedures}

Evaluations were carried out based on grain maturation stage, considering the dough stage reached at different times (Table 2). The plant was cut manually using a machete $\left(\right.$ Tramontina $^{\circledR}$ ) at a height of $10 \mathrm{~cm}$ above the soil, disregarding the lateral lines (considered bordering area) and 0.5 $\mathrm{m}$ from the extremities of the two central rows.
Plants from the usable area of each plot (2 central linear meters) were used for the evaluation of the following variables: number of tillers; plant height; percentages of leaf, stem, panicle, grain and dead material (dry or dead components); leaf:stem ratio (L:S); live:dead material ratio (LM:DM); total fresh and dry matter yields; and yields of leaf, stem, panicle, grain and dead material (in tha ${ }^{-1} \mathrm{DM}$ ).

Table 2

Sorghum hybrid cultivation period

\begin{tabular}{lccc}
\hline Hybrid & Planting & Harvest & Age (days) \\
\hline 9929036, 9929030, 9929026, 12F042226 & $01 / 10 / 2014$ & $04 / 26 / 2014$ & 106 \\
\hline $\begin{array}{l}\text { 12F042224, 12F042150, FEPAGRO18, FEPAGRO19, } \\
\text { FEPAGRO11, 9929012, 947216, 947030, 947072, 947252 }\end{array}$ & $01 / 10 / 2014$ & $05 / 08 / 2014$ & \multirow{2}{*}{118} \\
\hline 947254, 1141572, 12F042066, BRS Ponta Negra & $01 / 10 / 2014$ & $05 / 14 / 2014$ & 124 \\
\hline SF11, PROG134IPA, 1141570, 1141562, BRS506 & $01 / 10 / 2014$ & $05 / 17 / 2014$ & 127 \\
\hline SF15, SF25 & $01 / 10 / 2014$ & $05 / 24 / 2014$ & 134 \\
\hline
\end{tabular}

Number of tillers per linear meter was calculated based on the average of the total number of tillers in the usable plot area. Height was considered the average height of five plants chosen at random, measured with a steel measuring tape. The plants present within the usable area were harvested and weighed on a digital scale, which revealed the fresh mass.
For the morphological traits, two plants were collected from the usable plot area and separated into leaves, stems, dead mass and panicles, which were weighed individually to determine fresh weight. The samples were then dried in a forced-air oven at 55 ${ }^{\circ} \mathrm{C}$ for $72 \mathrm{~h}$ to determine the dry weight, as proposed by the Association of Official Analytical Chemists [AOAC] (1990). Subsequently, the yield of each morphological component (in $\mathrm{t} \mathrm{ha}^{-1}$ ) was quantified. 


\section{Chemical composition}

The surplus plants were processed through a stationary forage chopper (GT-2000L, Garthen ${ }^{\circledR}$ ) to particles of approximately $2 \mathrm{~cm}$. Composite samples of approximately $400 \mathrm{~g}$ of the material were collected and packed in paper bags (Semi Kraft $3 \mathrm{k}$ $\mathrm{g}^{-1}$, SamPlastic $\left.{ }^{\circledR}\right)$ to be pre-dried in a forced-air oven at $55{ }^{\circ} \mathrm{C}$ for $72 \mathrm{~h}$. Next, the samples were ground through a knife mill $\left(\right.$ Tecnal $\left.^{\circledR}\right)$, using a 1-mm sieve, and packed in 250-mL $\left(\right.$ Copobras $\left.^{\circledR}\right)$ containers with lid. Methods described in AOAC (1990) were used to determine the contents of dry matter (DM; in an oven without forced air circulation, at $105{ }^{\circ} \mathrm{C}$, method no. 934.01), crude protein (CP; using a nitrogen distillation apparatus - Kjeldahl method, method no. 920.87), ether extract (EE; using a Goldfish instrument, method no. 920.39), and neutral (NDF) and acid (ADF) detergent fiber [using $\alpha$-amylase, by the methods proposed by Van Soest, Robertson and Lewis (1991)]

\section{Model and statistical analysis}

The following statistical model was used:

$$
\mathrm{Y}_{\mathrm{ijk}}=\mu+\mathrm{H}_{\mathrm{i}}+\mathrm{B}_{\mathrm{j}}+\mathrm{e}_{\mathrm{ijk}} \text {, }
$$

where $\mathrm{Y}_{\mathrm{ijk}}=$ value obtained in plot $\mathrm{k}$ of treatment $\mathrm{i}$ in block $\mathrm{j} ; \mu=$ overall mean; $\mathrm{H}_{\mathrm{i}}=$ effect of observed hybrid $\mathrm{i} ; \mathrm{B}_{\mathrm{j}}=$ effect of block $\mathrm{j}$; and $\mathrm{e}_{\mathrm{ijk}}=$ residual effect of hybrids and blocks.

Data were subjected to analysis of variance and analyzed by the Scott-Knott procedure at the $5 \%$ significance level using SISVAR software (Computer Statistical Analysis System) (Ferreira, 2011).

\section{Results}

The hybrids differed $(\mathrm{P}<0.001)$ in terms of number of tillers per linear meter and plant height (cm) (Table 3). Number of tillers ranged from 5.17 to 30.83 per linear meter. For this variable, hybrids 9929036, 9929030, 12F042224, 12F042150, 9929012, 9929026, 947216, 947254, 947252, SF11, SF25, PROG134IPA, 1141572, 12F042226, 1141570,1141562 stood out, producing 16.00 to 30.83 tillers per linear meter.

Plant height ranged from 88.00 to $240.07 \mathrm{~cm}$. Similar means were separated into five groups: three hybrids showed an average height of 214.53, 229.00 and $240.07 \mathrm{~cm}$, which were the tallest (SF15, SF25 and PROG134IPA, respectively); six varied from 172.00 to $199.00 \mathrm{~cm}$ (FEPAGRO19, 1141572, 12F042150, 1141570, 1141562 and SF11); eight between 143.53 and $165.53 \mathrm{~cm}$ (947216, 12F042224, 947254, BRS Ponta Negra, FEPAGRO18, FEPAGRO11, BRS506 and 12F042066); six between 121.67 and $137.00 \mathrm{~cm}$; and, lastly, hybrids 12F042226, 947030, 9929012 , 9929036, 947252, 947072 and 9929030 and 9929026 were the shortest, ranging from 88.00 to $92.20 \mathrm{~cm}$, respectively.

There was a significant effect on the morphological characterization $(\mathrm{P}<0.001)$ for the percentages of stem, panicle and grain and LM:DM ratio, which ranged from 2.36 to $16.55 \%, 31.39$ to $86.21 \%, 1.61$ to $43.81 \%, 0.0$ to $36.30 \%$ and 0.03 to 0.57 , respectively (Table 4).

The highest stem percentages were obtained by hybrids BRS506, SF15, SF25, SF11 and PROG134IPA (86.21, 85.19, 83.95, 83.13 and $78.19 \%$, respectively) (Table 4). No effect was observed for dead material $(P=0.115)$ or LM:DM ratio $(\mathrm{P}=0.135)$. The highest grain yields were shown by hybrids 9929030, 12F042224, 12F042150, FEPAGRO18, FEPAGRO19, FEPAGRO11, 9929012, 9929026, 947216, 947030, 947254, 947072, 947252, 1141572, 12F042066, 12F042226, 1141562 and BRS Ponta Negra with values ranging from 13.96 to $36.30 \%$. 
Table 3

Number of tillers and plant height of sorghum hybrids

\begin{tabular}{lcc}
\hline Hybrid & Number of tillers & Plant height $(\mathrm{cm})$ \\
\hline 9929036 & $19.50 \mathrm{a}$ & $130.40 \mathrm{~d}$ \\
9929030 & $24.67 \mathrm{a}$ & $88.00 \mathrm{e}$ \\
12 F042224 & $18.17 \mathrm{a}$ & $145.27 \mathrm{c}$ \\
12 F042150 & $20.33 \mathrm{a}$ & $191.27 \mathrm{~b}$ \\
FEPAGRO18 & $7.17 \mathrm{~b}$ & $149.87 \mathrm{c}$ \\
FEPAGRO19 & $5.17 \mathrm{~b}$ & $172.00 \mathrm{~b}$ \\
FEPAGRO11 & $8.17 \mathrm{~b}$ & $149.93 \mathrm{c}$ \\
9929012 & $22.33 \mathrm{a}$ & $128.73 \mathrm{~d}$ \\
9929026 & $16.33 \mathrm{a}$ & $92.20 \mathrm{e}$ \\
947216 & $22.83 \mathrm{a}$ & $143.53 \mathrm{c}$ \\
947030 & $11.67 \mathrm{~b}$ & $123.87 \mathrm{~d}$ \\
947254 & $22.50 \mathrm{a}$ & $146.67 \mathrm{c}$ \\
947072 & $12.33 \mathrm{~b}$ & $137.00 \mathrm{~d}$ \\
947252 & $20.67 \mathrm{a}$ & $132.33 \mathrm{~d}$ \\
SF15 & $10.83 \mathrm{~b}$ & $214.53 \mathrm{a}$ \\
SF11 & $16.00 \mathrm{a}$ & $199.00 \mathrm{~b}$ \\
SF25 & $20.17 \mathrm{a}$ & $240.07 \mathrm{a}$ \\
PROG134IPA & $30.83 \mathrm{a}$ & $229.00 \mathrm{a}$ \\
1141572 & $16.17 \mathrm{a}$ & $186.20 \mathrm{~b}$ \\
12 F042066 & $13.67 \mathrm{~b}$ & $165.53 \mathrm{c}$ \\
12 F042226 & $17.83 \mathrm{a}$ & $121.67 \mathrm{~d}$ \\
1141570 & $17.83 \mathrm{a}$ & $193.40 \mathrm{~b}$ \\
BRS506 & $21.17 \mathrm{a}$ & $198.67 \mathrm{~b}$ \\
BRS Ponta Negra & $10.17 \mathrm{~b}$ & $162.20 \mathrm{c}$ \\
CV\% & $8.17 \mathrm{~b}$ & $148.47 \mathrm{c}$ \\
\hline P-value & 29.98 & 10.08 \\
\hline & $<0.001$ & $<0.001$ \\
\hline
\end{tabular}

${ }^{1}$ Means followed by common letters in the column do not differ according to the Scott-Knott procedure at the significance level of $0.05 . \mathrm{CV}=$ coefficient of variation.

Total fresh matter yield varied $(\mathrm{P}<0.001)$, with hybrids SF15, SF11 and 12F042150 exhibiting the highest values $\left(93.95,82.61\right.$ and $79.27 \mathrm{t} \mathrm{ha}^{-1}$, respectively) (Table 5), while SF15 was superior for total dry matter yield $\left(35.33 \mathrm{tha}^{-1}\right)$.

There was an effect $(P=0.002)$ for the quantification of the morphological components, with leaf yield ranging from 0.37 to $2.46 \mathrm{t} \mathrm{ha}^{-}$ 1. For stem yield $(\mathrm{P}<0.001)$, the highest mean was obtained by SF15 (30.08 $\left.\mathrm{t} \mathrm{ha}^{-1}\right)$, while 47072 produced the highest yields of panicle and grain $(\mathrm{P}$ $<0.001$ ) (12.21 and 10.14 t ha ${ }^{-1}$, respectively). Dead material yield did not differ $(\mathrm{P}=0.150)$ between the hybrids (Table 5).

Differences were detected $(\mathrm{P}<0.05)$ in chemical composition of sorghum for the DM, CP, EE, and NDF contents, which varied from 26.83 to $42.74 \%$, 4.98 to $8.64 \%, 0.89$ to $1.78 \%$ and 55.18 to $78.19 \%$, respectively (Table 6). There was no effect $(\mathrm{P}=$ 0.140) for ADF. 
Table 4

Morphological characterization of sorghum hybrids on a dry matter basis

\begin{tabular}{|c|c|c|c|c|c|c|c|}
\hline \multirow{3}{*}{ Hybrid } & \multicolumn{7}{|c|}{ Trait } \\
\hline & \multicolumn{5}{|c|}{ Component $(\%)$} & \multicolumn{2}{|c|}{ Ratio } \\
\hline & Leaf & Stem & Panicle & Grains & Dead material & $\mathrm{L}: \mathrm{S}$ & DM:LM \\
\hline 9929036 & $11.01 \mathrm{~b}$ & $69.46 \mathrm{~b}$ & $10.22 \mathrm{~b}$ & $0.00 \mathrm{~b}$ & 9.31 & $0.16 \mathrm{c}$ & 11.00 \\
\hline 9929030 & $16.55 \mathrm{a}$ & $31.39 \mathrm{e}$ & $31.63 \mathrm{a}$ & $24.18 \mathrm{a}$ & 20.42 & $0.57 \mathrm{a}$ & 10.39 \\
\hline $12 \mathrm{~F} 042224$ & $5.01 \mathrm{c}$ & $49.71 \mathrm{~d}$ & $41.64 \mathrm{a}$ & $35.67 \mathrm{a}$ & 3.65 & $0.10 \mathrm{c}$ & 33.28 \\
\hline 12F042150 & $5.09 \mathrm{c}$ & $62.25 \mathrm{c}$ & $24.62 \mathrm{a}$ & $16.92 \mathrm{a}$ & 8.05 & $0.09 \mathrm{c}$ & 13.08 \\
\hline FEPAGRO18 & $4.99 \mathrm{c}$ & $65.18 \mathrm{c}$ & $25.89 \mathrm{a}$ & $21.51 \mathrm{a}$ & 3.94 & $0.08 \mathrm{c}$ & 32.47 \\
\hline FEPAGRO19 & $2.92 \mathrm{c}$ & $72.81 \mathrm{~b}$ & $20.66 \mathrm{a}$ & $17.27 \mathrm{a}$ & 3.61 & $0.04 \mathrm{c}$ & 32.48 \\
\hline FEPAGRO11 & $5.79 \mathrm{c}$ & $57.48 \mathrm{c}$ & $32.13 a$ & $27.00 \mathrm{a}$ & 4.60 & $0.10 \mathrm{c}$ & 40.58 \\
\hline 9929012 & $9.28 \mathrm{~b}$ & $51.58 \mathrm{~d}$ & $26.75 \mathrm{a}$ & $21.39 \mathrm{a}$ & 12.39 & $0.19 \mathrm{c}$ & 22.06 \\
\hline 9929026 & $14.82 \mathrm{a}$ & $43.58 \mathrm{~d}$ & $25.76 \mathrm{a}$ & $20.17 \mathrm{a}$ & 15.84 & $0.34 \mathrm{~b}$ & 6.83 \\
\hline 947216 & $8.42 b$ & $52.83 \mathrm{~d}$ & $21.39 \mathrm{a}$ & $14.85 \mathrm{a}$ & 17.37 & $0.18 \mathrm{c}$ & 9.29 \\
\hline 947030 & $2.94 \mathrm{c}$ & $59.09 \mathrm{c}$ & $29.91 \mathrm{a}$ & $25.99 \mathrm{a}$ & 8.07 & $0.05 \mathrm{c}$ & 11.83 \\
\hline 947254 & $2.71 \mathrm{c}$ & $62.12 \mathrm{c}$ & $30.24 \mathrm{a}$ & $26.46 \mathrm{a}$ & 4.93 & $0.04 \mathrm{c}$ & 19.38 \\
\hline 947072 & $4.01 \mathrm{c}$ & $44.09 \mathrm{~d}$ & $43.81 \mathrm{a}$ & $36.30 \mathrm{a}$ & 8.09 & $0.09 \mathrm{c}$ & 12.53 \\
\hline 947252 & $6.49 \mathrm{c}$ & $57.65 \mathrm{c}$ & $28.85 \mathrm{a}$ & $20.85 \mathrm{a}$ & 7.01 & $0.12 \mathrm{c}$ & 14.00 \\
\hline SF15 & $3.62 \mathrm{c}$ & $85.19 \mathrm{a}$ & $6.02 \mathrm{~b}$ & $2.05 b$ & 5.16 & $0.04 \mathrm{c}$ & 23.64 \\
\hline SF11 & $8.51 \mathrm{~b}$ & $83.13 \mathrm{a}$ & $2.13 b$ & $0.00 \mathrm{~b}$ & 6.23 & $0.10 \mathrm{c}$ & 15.46 \\
\hline SF25 & $4.61 \mathrm{c}$ & $83.95 \mathrm{a}$ & $6.66 \mathrm{~b}$ & $0.84 \mathrm{~b}$ & 4.79 & $0.06 \mathrm{c}$ & 21.54 \\
\hline PROG134IPA & $11.61 \mathrm{~b}$ & $78.19 \mathrm{a}$ & $1.61 \mathrm{~b}$ & $0.00 \mathrm{~b}$ & 8.58 & $0.15 \mathrm{c}$ & 12.27 \\
\hline 1141572 & $2.88 \mathrm{c}$ & $69.05 \mathrm{~b}$ & $21.62 \mathrm{a}$ & $17.05 \mathrm{a}$ & 6.45 & $0.04 \mathrm{c}$ & 15.27 \\
\hline $12 \mathrm{~F} 042066$ & $4.12 \mathrm{c}$ & $56.40 \mathrm{c}$ & $32.85 \mathrm{a}$ & $29.67 \mathrm{a}$ & 6.63 & $0.07 \mathrm{c}$ & 15.33 \\
\hline $12 \mathrm{~F} 042226$ & $9.83 b$ & $50.35 \mathrm{~d}$ & $32.21 \mathrm{a}$ & $14.69 \mathrm{a}$ & 7.60 & $0.19 \mathrm{c}$ & 13.58 \\
\hline 1141570 & $2.36 \mathrm{c}$ & $72.59 \mathrm{~b}$ & $15.96 \mathrm{~b}$ & $10.65 b$ & 9.09 & $0.03 \mathrm{c}$ & 11.59 \\
\hline 1141562 & $7.63 b$ & $60.03 \mathrm{c}$ & $18.16 \mathrm{a}$ & $13.96 \mathrm{a}$ & 14.18 & $0.13 \mathrm{c}$ & 8.52 \\
\hline BRS506 & $4.92 \mathrm{c}$ & $86.21 \mathrm{a}$ & $3.96 \mathrm{~b}$ & $0.00 \mathrm{~b}$ & 4.90 & $0.06 \mathrm{c}$ & 20.33 \\
\hline BRS Ponta Negra & $7.95 \mathrm{~b}$ & $63.05 \mathrm{c}$ & $21.52 \mathrm{a}$ & $17.87 \mathrm{a}$ & 7.48 & $0.12 \mathrm{c}$ & 12.72 \\
\hline $\mathrm{CV} \%$ & 50.15 & 12.10 & 41.61 & 55.20 & 75.38 & 76.23 & 72.28 \\
\hline P-value & $<0.001$ & $<0.001$ & $<0.001$ & $<0.001$ & 0.115 & $<0.001$ & 0.135 \\
\hline
\end{tabular}

${ }^{1}$ Means followed by common letters in the column do not differ according to the Scott-Knott procedure at the significance level of 0.05. L:S = leaf:stem; $\mathrm{LM}: \mathrm{DM}=$ live:dead material; $\mathrm{CV}=$ coefficient of variation. 
Table 5

Total fresh and dry matter yields and yields of morphological components of sorghum hybrids

\begin{tabular}{|c|c|c|c|c|c|c|c|}
\hline \multirow[t]{2}{*}{ Hybrid } & \multicolumn{2}{|c|}{ Total yield $\left(\mathrm{t} \mathrm{ha}^{-1}\right)$} & \multicolumn{5}{|c|}{ Yield $\left(\mathrm{t} \mathrm{ha}^{-1} \mathrm{DM}\right)$} \\
\hline & Fresh matter & Dry matter & Leaf & Stem & Panicle & Grain & Dead material \\
\hline 9929036 & $67.20 \mathrm{~b}$ & $20.00 \mathrm{c}$ & $2.11 \mathrm{a}$ & $13.91 \mathrm{c}$ & $1.97 \mathrm{c}$ & $0.00 \mathrm{~d}$ & 3.08 \\
\hline 9929030 & $41.88 \mathrm{c}$ & $16.30 \mathrm{c}$ & $2.46 \mathrm{a}$ & $5.28 \mathrm{~d}$ & $5.48 b$ & $4.28 \mathrm{~b}$ & 2.02 \\
\hline $12 \mathrm{~F} 042224$ & $45.99 \mathrm{c}$ & $15.02 \mathrm{c}$ & $0.76 \mathrm{~b}$ & $7.32 \mathrm{~d}$ & $6.36 \mathrm{~b}$ & $5.45 \mathrm{~b}$ & 0.58 \\
\hline $12 \mathrm{~F} 042150$ & $79.27 \mathrm{a}$ & $25.57 b$ & $1.21 \mathrm{~b}$ & $16.01 \mathrm{c}$ & $6.29 b$ & $4.35 \mathrm{~b}$ & 2.05 \\
\hline FEPAGRO 18 & $32.19 \mathrm{c}$ & $10.67 \mathrm{c}$ & $0.53 b$ & $6.95 \mathrm{~d}$ & $2.81 \mathrm{c}$ & $2.34 \mathrm{c}$ & 0.38 \\
\hline FEPAGRO 19 & $43.88 \mathrm{c}$ & $15.41 \mathrm{c}$ & $0.43 b$ & $11.13 \mathrm{c}$ & $3.32 \mathrm{c}$ & $2.81 \mathrm{c}$ & 0.54 \\
\hline FEPAGRO 11 & $41.37 \mathrm{c}$ & $14.66 \mathrm{c}$ & $0.86 \mathrm{~b}$ & $8.62 \mathrm{~d}$ & $4.37 \mathrm{~b}$ & $3.64 \mathrm{~b}$ & 0.81 \\
\hline 9929012 & $39.49 \mathrm{c}$ & $16.47 \mathrm{c}$ & $1.56 \mathrm{a}$ & $8.36 \mathrm{~d}$ & $4.51 \mathrm{~b}$ & $3.61 \mathrm{~b}$ & 2.03 \\
\hline 9929026 & $38.92 \mathrm{c}$ & $11.95 \mathrm{c}$ & $1.75 \mathrm{a}$ & $5.12 d$ & $3.31 \mathrm{c}$ & $2.63 \mathrm{c}$ & 1.78 \\
\hline 947216 & $47.20 \mathrm{c}$ & $18.63 \mathrm{c}$ & $1.58 \mathrm{a}$ & $9.97 \mathrm{~d}$ & $4.15 \mathrm{~b}$ & $2.86 \mathrm{c}$ & 2.92 \\
\hline 947030 & $35.50 \mathrm{c}$ & $14.28 \mathrm{c}$ & $0.37 \mathrm{~b}$ & $8.02 \mathrm{~d}$ & $4.69 \mathrm{~b}$ & $4.12 b$ & 1.20 \\
\hline 947254 & $37.99 \mathrm{c}$ & $16.98 \mathrm{c}$ & $0.46 \mathrm{~b}$ & $10.55 \mathrm{~d}$ & $5.13 b$ & $4.50 \mathrm{~b}$ & 0.83 \\
\hline 947072 & $61.77 \mathrm{~b}$ & $27.87 \mathrm{~b}$ & $1.07 \mathrm{~b}$ & $12.27 \mathrm{c}$ & $12.21 \mathrm{a}$ & $10.14 \mathrm{a}$ & 2.32 \\
\hline 947252 & $43.88 \mathrm{c}$ & $18.47 \mathrm{c}$ & $1.15 b$ & $10.46 \mathrm{~d}$ & $5.51 \mathrm{~b}$ & $4.05 \mathrm{~b}$ & 1.34 \\
\hline SF15 & $93.95 \mathrm{a}$ & $35.33 \mathrm{a}$ & $1.27 \mathrm{~b}$ & $30.08 \mathrm{a}$ & $2.12 \mathrm{c}$ & $0.71 \mathrm{~d}$ & 1.85 \\
\hline SF11 & $82.61 \mathrm{a}$ & $23.86 \mathrm{~b}$ & $1.84 \mathrm{a}$ & $19.94 \mathrm{~b}$ & $0.54 \mathrm{c}$ & $0.00 \mathrm{~d}$ & 1.53 \\
\hline SF25 & $68.08 \mathrm{~b}$ & $25.91 b$ & $1.13 b$ & $21.95 b$ & $1.65 \mathrm{c}$ & $0.21 \mathrm{~d}$ & 1.17 \\
\hline PROG134IPA & $60.51 b$ & $18.36 \mathrm{c}$ & $2.14 \mathrm{a}$ & $14.36 \mathrm{c}$ & $0.29 \mathrm{c}$ & $0.00 \mathrm{~d}$ & 1.57 \\
\hline 1141572 & $60.41 b$ & $22.19 b$ & $0.62 b$ & $15.30 \mathrm{c}$ & $4.81 \mathrm{~b}$ & $3.79 b$ & 1.44 \\
\hline $12 \mathrm{~F} 042066$ & $35.37 \mathrm{c}$ & $16.28 \mathrm{c}$ & $0.72 b$ & $9.27 \mathrm{~d}$ & $5.18 b$ & $4.67 \mathrm{~b}$ & 1.11 \\
\hline $12 \mathrm{~F} 042226$ & $46.60 \mathrm{c}$ & $14.23 \mathrm{c}$ & $1.39 \mathrm{a}$ & $6.96 \mathrm{~d}$ & $4.77 \mathrm{~b}$ & $2.01 \mathrm{c}$ & 1.10 \\
\hline 1141570 & $60.99 b$ & $21.26 \mathrm{~b}$ & $0.58 b$ & $15.98 \mathrm{c}$ & $2.94 \mathrm{c}$ & $1.81 \mathrm{c}$ & 1.76 \\
\hline 1141562 & $51.48 \mathrm{c}$ & $21.43 b$ & $1.61 \mathrm{a}$ & $13.20 \mathrm{c}$ & $3.94 b$ & $3.05 \mathrm{c}$ & 2.68 \\
\hline BRS506 & $45.67 \mathrm{c}$ & $13.65 \mathrm{c}$ & $0.67 b$ & $11.78 \mathrm{c}$ & $0.54 \mathrm{c}$ & $0.00 \mathrm{~d}$ & 0.67 \\
\hline BRS Ponta Negra & $35.65 \mathrm{c}$ & $11.91 \mathrm{c}$ & $0.81 \mathrm{~b}$ & $7.42 \mathrm{~d}$ & $2.76 \mathrm{c}$ & $2.32 \mathrm{c}$ & 0.92 \\
\hline $\mathrm{CV} \%$ & 24.06 & 26.61 & 54.62 & 30.14 & 48.98 & 60.94 & 71.55 \\
\hline P-value & $<0.001$ & 0.025 & 0.002 & $<0.001$ & $<0.001$ & $<0.001$ & 0.150 \\
\hline
\end{tabular}

${ }^{1}$ Means followed by common letters in the column do not differ according to the Scott-Knott procedure at the significance level of 0.05. $\mathrm{DM}=$ dry matter; $\mathrm{CV}=$ coefficient of variation. 
Table 6 Chemical composition of sorghum hybrids

\begin{tabular}{lccccc}
\hline Hybrid & DM\% & CP\% & EE\% & NDF\% & ADF\% \\
\hline 9929036 & $26.83 \mathrm{c}$ & $5.67 \mathrm{~b}$ & $1.33 \mathrm{~b}$ & $66.13 \mathrm{~b}$ & 40.73 \\
9929030 & $34.97 \mathrm{~b}$ & $7.93 \mathrm{a}$ & $1.72 \mathrm{a}$ & $71.26 \mathrm{~b}$ & 41.11 \\
12 F042224 & $30.32 \mathrm{c}$ & $6.37 \mathrm{~b}$ & $1.78 \mathrm{a}$ & $69.60 \mathrm{~b}$ & 41.17 \\
12 F042150 & $30.30 \mathrm{c}$ & $6.34 \mathrm{~b}$ & $1.36 \mathrm{~b}$ & $74.40 \mathrm{a}$ & 36.52 \\
FEPAGRO18 & $30.81 \mathrm{c}$ & $6.57 \mathrm{a}$ & $1.59 \mathrm{a}$ & $71.40 \mathrm{~b}$ & 36.74 \\
FEPAGRO19 & $32.94 \mathrm{c}$ & $5.50 \mathrm{~b}$ & $1.44 \mathrm{~b}$ & $74.00 \mathrm{a}$ & 37.38 \\
FEPAGRO11 & $32.20 \mathrm{c}$ & $6.75 \mathrm{a}$ & $1.69 \mathrm{a}$ & $76.46 \mathrm{a}$ & 38.46 \\
9929012 & $38.55 \mathrm{a}$ & $5.91 \mathrm{~b}$ & $1.50 \mathrm{~b}$ & $77.67 \mathrm{a}$ & 42.20 \\
9929026 & $28.38 \mathrm{c}$ & $8.64 \mathrm{a}$ & $1.58 \mathrm{a}$ & $77.53 \mathrm{a}$ & 40.00 \\
947216 & $36.71 \mathrm{~b}$ & $5.80 \mathrm{~b}$ & $1.40 \mathrm{~b}$ & $77.67 \mathrm{a}$ & 35.16 \\
947030 & $35.91 \mathrm{~b}$ & $6.68 \mathrm{~b}$ & $1.32 \mathrm{~b}$ & $69.67 \mathrm{~b}$ & 38.95 \\
947254 & $41.46 \mathrm{a}$ & $6.25 \mathrm{~b}$ & $1.45 \mathrm{~b}$ & $78.07 \mathrm{a}$ & 40.41 \\
947072 & $42.31 \mathrm{a}$ & $6.97 \mathrm{a}$ & $1.62 \mathrm{a}$ & $70.92 \mathrm{~b}$ & 42.16 \\
947252 & $39.43 \mathrm{a}$ & $6.55 \mathrm{a}$ & $1.43 \mathrm{~b}$ & $78.19 \mathrm{a}$ & 43.44 \\
SF15 & $34.26 \mathrm{~b}$ & $5.36 \mathrm{~b}$ & $1.00 \mathrm{c}$ & $67.92 \mathrm{~b}$ & 42.19 \\
SF11 & $26.86 \mathrm{c}$ & $6.32 \mathrm{~b}$ & $1.07 \mathrm{c}$ & $68.22 \mathrm{~b}$ & 37.03 \\
SF25 & $34.76 \mathrm{~b}$ & $5.32 \mathrm{~b}$ & $0.89 \mathrm{c}$ & $68.90 \mathrm{~b}$ & 38.31 \\
PROG134IPA & $27.81 \mathrm{c}$ & $5.86 \mathrm{~b}$ & $1.41 \mathrm{~b}$ & $69.47 \mathrm{~b}$ & 41.44 \\
1141572 & $33.54 \mathrm{c}$ & $4.98 \mathrm{~b}$ & $1.43 \mathrm{~b}$ & $73.82 \mathrm{a}$ & 42.62 \\
12 F042066 & $42.74 \mathrm{a}$ & $6.14 \mathrm{~b}$ & $1.28 \mathrm{~b}$ & $76.96 \mathrm{a}$ & 40.63 \\
12 F042226 & $27.72 \mathrm{c}$ & $6.93 \mathrm{a}$ & $1.51 \mathrm{~b}$ & $67.85 \mathrm{~b}$ & 38.74 \\
1141570 & $32.16 \mathrm{c}$ & $5.47 \mathrm{~b}$ & $1.63 \mathrm{a}$ & $68.64 \mathrm{~b}$ & 40.27 \\
1141562 & $39.29 \mathrm{a}$ & $5.15 \mathrm{~b}$ & $1.32 \mathrm{~b}$ & $65.00 \mathrm{~b}$ & 39.70 \\
BRS506 & $27.32 \mathrm{c}$ & $5.45 \mathrm{~b}$ & $1.07 \mathrm{c}$ & $55.18 \mathrm{c}$ & 30.24 \\
BRS Ponta Negra & $30.00 \mathrm{c}$ & $7.61 \mathrm{a}$ & $1.67 \mathrm{a}$ & $68.04 \mathrm{~b}$ & 37.57 \\
\hline${ }^{6}$ CV\% & 11.63 & 15.16 & 9.46 & 6.60 & 9.65 \\
\hline P-value & $<0.001$ & $<0.001$ & $<0.001$ & 0.018 & 0.140 \\
\hline
\end{tabular}

${ }^{1}$ Means followed by common letters in the column do not differ according to the Scott-Knott procedure at the significance level of $0.05 . \mathrm{DM}=$ dry matter; $\mathrm{CP}=$ crude protein; $\mathrm{EE}=$ ether extract; $\mathrm{NDF}=$ neutral detergent fiber; $\mathrm{ADF}=$ acid detergent fiber; $\mathrm{CV}=$ coefficient of variation .

\section{Discussion}

The sorghum crop can usually be harvested twice. However, the lack of rain due to the earlier end of the rainy period after the first harvest did not allow for regrowth, in the present experiment (Figure 1).

The use of hybrids with large plant populations may result in a lesser need for cultivation treatments during the development of the crop. Plants with larger tiller populations can occupy larger spaces, thereby increasing shading, which then hinders the development of undesirable plants. When this trait is associated with elevated plant height and low lodging, total dry matter yield tends to increase.

According to Pereira and Rodrigues (2015), dualpurpose or medium-height sorghum varieties have a 
grain percentage of 30 to $40 \%$ and grow 2 to $2.5 \mathrm{~m}$. In this respect, the hybrids whose height was within the said range were SF15, SF25 and PROG134IPA. Hybrids 9929036, 12F042224, FEPAGRO18, FEPAGRO11, 9929012, 947216, 947030, 947254, 947072, 947252, 12F042226 and BRS Ponta Negra, in turn, fit into the grain category. Pereira and Rodrigues (2015) classified low to medium-sized plants as those with a height between 1.20 and 1.50 $\mathrm{m}$, since their main product is the grain found on the panicles, which can be harvested when dry.

As stated by Neumann et al. (2002), plant height is a trait that regularly determines its productive potential in dry and fresh matter per hectare. Therefore, the components of dry matter yield in sorghum are usually related to plant height. However, taller sorghum plants have higher lodging rates and tend to be less early-developing, which may constitute an inadequate characteristic for regions with short rainfall periods and thus render off-season planting unfeasible, since its growth is slower.

The lowest mean heights were found in hybrids 9929030 and 9929026 (88.00 and 92.20 $\mathrm{cm}$, respectively) (Table 3), which are among the earliest hybrids harvested at 106 days after planting (Table 2). Like 9929036 and 12F042226, they may be used in the sorghum off-season for grain or silage production or even serve as straw for direct planting. Mahmood and Honermeier (2012) asserted that short-cycle sorghum serves for crop rotation or subsequent crops of main grain production, or may also be used in integration systems.

Hybrids 9929030 and 9929026 showed the highest leaf percentages (16.55 and 14.82\%), whereas the others were arranged in two groups with lower means, which ranged from 7.63 to $11.61 \%$ and 2.36 to $6.49 \%$ (Table 4 ). These findings differ from those reported by Xiao-Ping, Jin-Feng, Cui-Ping and Acharya (2011), who evaluated morphological components and observed leaf percentages of 8.94 to $28.81 \%$. This great variation may be due to the better adaptation of those hybrids, as described by $\mathrm{T}$. C. Silva et al. (2012), who studied the morphological components of sorghum and stated that adaptation to the soil and local climatic conditions allows for higher leaf percentages. However, leaves of $\mathrm{C}_{4}$ plants may lose less water due to the ability of their stomata to remain closed longer, as a consequence of the $\mathrm{CO}_{2}$ pump that facilitates photosynthetic rate even under low $\mathrm{CO}_{2}$ conditions in the intracellular spaces (Döring, Streubel, Bräutigam, \& Gowik, 2016).

Neves et al. (2014) obtained a high correlation between plant height and stem percentage in SF15. In the current study, this was observed for SF15, SF25 and PROG134IPA, which were taller (Table 3 ) and had high stem percentages (Table 4).

Ikanovic et al. (2010) found a positive correlation between leaf mass and stem mass. R. Silva, Santos, Tabosa, Gomes and Almeida (2012), in turn, suggested that sorghum plants with a high grain yield are unsuitable for silage making, but mostly indicated for use as forage.

A higher participation of panicle in the sorghum plant production may result in a silage of higher nutritional value (T. C. Silva et al., 2011). In the present experiment, some hybrids showed low percentages of this component (9929036, SF15, SF11, SF25, PROG134IPA, 1141570 and BRS506), which ranged from 1.61 to $15.96 \%$. These hybrids were found to have high stem percentages. Higher panicle percentages possibly translate into higher grain yields, which may explain the similar and low grain yields of those hybrids.

Hybrids 9929036, 12F042226 SF15 and SF25 BRS506 had low grain yields because of the action of birds, which consumed the grains from the first two when they were still green, and the last one when still in the grain dough stage, where it possibly presents low tannin contents. In the case of hybrids SF15, SF11, SF25 and PROG134IPA, these were harvested in a period during which they were already undergoing water stress, in the month 
of May (Figure 1), which corresponds to the end of the rainy period in the region where the study took place. As such, they had not fully produced their panicles and were beginning the senescence process. The fact that hybrids SF15 and SF25 were the latest-developing (Table 2) may explain their low panicle and grain yields.

The higher leaf percentage (16.55\%), and lower participation of stem $(31.39 \%)$ found in 9929030 may explain the higher LM:DM ratio shown by that hybrid (Table 4). This hybrid also had high percentages of panicle (31.63\%) and grain $(24.18 \%)$ and a short plant height $(88.00 \mathrm{~cm})$ (Table 3), which are typical traits of grain sorghum. Shorter plants tend to have higher LM:DM ratios, while the opposite occurs in taller plants. This phenomenon is due to the higher percentages of stem, which has high cell wall components, forming a supporting tissue, which in turn is influenced by plant age (McDonald et al., 2011).

Perazzo et al. (2013) evaluated sorghum hybrids and observed that SF 15 produced $10.77 \%$ of dead material, which was due to the greater acceleration of the cycle in response to the water deficit. Significant fresh mass values and low senescent mass values may explain the lack of effects (Table 4).

Rodrigues et al. (2006) evaluated the productive potential of hybrids and observed an herbage yield that ranged from 45.87 to $67.56 \mathrm{t} \mathrm{ha}^{-1}$. When aiming at silage production, sorghum varieties with high herbage mass yields are preferred, since this is a form of preserving fresh forage. The plant heights of 214.53, 199.00 and $191.27 \mathrm{~cm}$ (Table 3) and stem percentages of $85.19,83.13$ and $62.25 \%$ (Table 4) found in SF15, SF11 and 12F042150, respectively, may explain their outstanding fresh matter yield (Table 5).

The present results for DM yield (Table 5) reveal a great discrepancy, as also found by T. C. Silva et al. (2011), who evaluated the DM yield of 25 sorghum hybrids in the Agreste Paraibano region and fond values ranging from 7.67 to $20.94 \mathrm{t} \mathrm{ha}^{-1}$. Hybrid
SF15 had the highest DM yield (35.33 $\left.\mathrm{tha}^{-1}\right)$, while the others produced 10.67 to $27.87 \mathrm{t} \mathrm{DM} \mathrm{ha}^{-1}$. These mean values may be related to plant height and stem percentage.

Hybrids 9929036, 9929030, 9929012, 9929026, 947216, SF11, PROG134IPA, 12F042226 and 1141562 showed the highest leaf yields (1.39 to $2.46 \mathrm{t} \mathrm{h}^{-1}$ ). Leaves represent the plant fraction of greatest importance to animal nutrition and, as such, should be considered in the selection of sorghum (Table 5). Leaf area expansion may be gradually reduced with delayed flowering (Tazoe et al., 2016). Coupled with the diversity of the tested hybrids, this phenomenon may explain the discrepant values.

The stem typically contains a larger amount of fiber than the leaf, which directly affects plant yield. This allowed hybrid SF15 to have a higher stem yield than the other hybrids, whose mean values ranged from 5.28 to $19.94 \mathrm{t} \mathrm{ha}^{-1}$. Although the stem is a reserve of nutrients in the plant, it is also a great deposit of resistant structures such as lignin and cell wall, which affect its nutritional value (Van Soest, 1994).

Grain yield may be related to other traits. The higher the panicle yield, the higher grain yield tends to be. However, in this study, some hybrids with a longer cycle (SF15, SF11, SF25 and PROG134IPA) (Table 2) were chopped before the grain dough stage due to lack of rainfall, which compromised the plant's cycle, consequently influencing grain yield.

Grain yield in hybrids 9929036, 12F042226 and BRS506 was compromised by the action of birds. This indicates that they are not suitable for silage making, since the grain is important in obtaining high-quality silage and bird control is an unfeasible and oftentimes environmentally inappropriate practice. Those factors likely contributed to the low panicle and grain yields, which compromises the yield of the sorghum crop. R. Silva et al. (2012) stated that the panicle and whole-plant masses should be observed together for the different purposes evaluated in studies. 
Most hybrids were harvested in the dough grain stage, and because the plant tissues are not under an advanced state of senescence in that stage, dead material yield was not influenced (Table 5).

A DM content of 30 to $35 \%$ is recommended for silage production. Values below this range favor the fermentation of Clostridium spp., due to inadequate moisture contents (Table 6). Lima, Rangel, Urbano, Oliveira and Maciel (2014) reported that a high moisture content dilutes the acids produced by heterofermentative bacteria, causing a resistance to $\mathrm{pH}$ decline. Accordingly, the hybrids whose DM mean were within the said range were 9929030, 12F042224, 12F042150, FEPAGRO18, FEPAGRO19, FEPAGRO11, SF15, SF25, 1141572 , 1141570 and BRS Ponta Negra.

The highest CP values were found in hybrids 9929030, FEPAGRO18, FEPAGRO11, 9929026, 947072, 947252, 12F042226 and BRS Ponta Negra (6.55 to $8.64 \%$ ), which is most obviously due to their grain and leaf production. Simili, Lima, Medeiros, Paz and Reis (2014), found a CP content of $6.71 \%$ in the composition of sorghum 1P400. Mabelebele, Siwela, Gous and Iji (2015), in turn, found 8.1 to 9.5\% CP in grain sorghum.

The highest EE means were found in hybrids 9929030, 12F042224, FEPAGRO18, FEPAGRO11, 9929026, 947072, 1141570 and BRS Ponta Negra (Table 6). This result may be due to the leaf, panicle and grain components (Table 4), which have higher concentrations of EE. Similar findings were reported by Moraes, Jobim, Silva and Marquardt (2013) in four sorghum hybrids for silage (AG 2005E, XBS 60015, XBS 60451 and DowF305), where EE ranged from 1.44 to $2.36 \%$ in the whole plant, 2.51 to $3.54 \%$ in the leaf and 2.33 to $3.82 \%$ in the grain.

Chemical compositions may vary between hybrids depending on the age and morphological part of the plant (Mahmood \& Honermeier, 2012). In this regard, BRS506 showed the lowest average NDF content (55.18\%). Its stem was possibly thick, considering the stem percentage of $86.21 \%$
(Table 4), but this result may also be attributed to the cultivation time of 127 days (Table 2). Gomes, Pitombeira, Neiva and Cândido (2006) observed lower values in the same cultivar at 102 days of growth (78\% stem and $45.94 \% \mathrm{NDF})$.

Hybrids 9929036， 9929030， 12F042224, FEPAGRO18, 947030, 947072, SF15, SF11, SF25, PROG134IPA, 12F042226, 1141570, 1141562 and BRS Ponta Negra showed NDF contents ranging from 65.00 to $71.40 \%$, corroborating the results reported by Pedreira, Reis, Berchielli, Moreira and Coan (2003), who studied eight hybrids and found NDF values of 57 to $70.3 \%$. The highest NDF means $(73.82$ to $78.19 \%$ ) were found in hybrids 12F042150, FEPAGRO19, FEPAGRO11, 9929012, 9929026, 947216, 947254, 947252, 1141572 and 12F042066. High NDF concentrations can influence total DM intake, due to the limitation caused by rumen fill (Alves et al., 2016). Additionally, ADF is directly related to NDF, and intake is influenced when both components are present at high levels (Gomes, Pitombeira, Neiva, \& Cândido, 2006). However, the ADF values obtained in this study are close to the $40 \%$ considered adequate for ruminants (Van Soest, 1994).

\section{Conclusion}

Hybrids 9929030, 12F042224， 12F042150, FEPAGRO19, FEPAGRO11, 1141572 and 1141570 possess an agronomic profile and chemical composition in rainfed conditions compatible with silage production.

\section{References}

Alves, A. R., Pascoal, L. A. F., Cambuí, G. B., Trajano, J. S., Silva, C. M., \& Gois, G. C. (2016). Fibra para ruminantes: aspecto nutricional, metodológico e funcional. PUBVET, 10(7), 568-579. doi: 10.1590/1142e0e16dd9d3280d9ab0508c86149

Association of Official Analytical Chemists (1990). Official methods of analysis of the AOAC (15th ed.). Arlington: Virginea. 
Avelino, P. M., Neiva, J. M. N., Araújo, V. L., Alexandrino, E., Bomfim, M. A. D., \& Restle, J. (2011). Composição bromatológica de silagens de híbridos de sorgo cultivados em diferentes densidades de plantas. Revista Ciência Agronômica, 42(1), 208215. doi: 10.1590/S1806-66902011000100026

Cândido, M. J. D., Obeid, J. Á., Pereira, O. D., Cecon, P. R., Queiroz, A. C., Paulino, M. F., \& Gontijo, M. M., Neto. (2002). Valor nutritivo de silagens de híbridos de sorgo (Sorghum bicolor (L.) Moench) sob doses crescentes de adubação. Revista Brasileira de Zootecnia, 31(1), 20-29. doi: 10.1590/S151635982002000100003

Cunha, E. E., \& Lima, J. M. P. (2010). Caracterização de genótipos e estimativa de parâmetros genéticos de características produtivas de sorgo forrageiro. Revista Brasileira de Zootecnia, 39(4), 701-706. doi: 10.1590/S1516-35982010000400002

Döring, F., Streubel, M., Bräutigam, A., \& Gowik, U. (2016). Most photorespiratory genes are preferentially expressed in the bundle sheath cells of the $\mathrm{C}_{4}$ grass Sorghum bicolor. Journal of Experimental Botany, 67(10), 3053-3064. doi: 10.1093/jxb/erw041

Ferreira, D. F. (2011). Sisvar: computer statistical analysis system. Ciência e Agrotecnologia, 35(6), 1039-1042. doi: 10.1590/S1413-70542011000600001

Gomes, S. O., Pitombeira, J. B., Neiva, J. N. M., \& Cândido, M. J. D. (2006). Comportamento agronômico e composição químico-bromatológico de cultivares de sorgo forrageiro no Estado do Ceará. Revista Ciência Agronômica, 37(2), 221-227.

Ikanovic, J., Glamoclija, D., Maletic, R., Jankovic, S., Tabakovic, M., \& Zivanovic, L. (2010). The genotype traits of forage sorghum, sudan grass and their interspecies hybrid in the conditions of intensive nitrogen nutrition. Genetika, 42(2), 349358. doi: 10.2298/GENSR1002349I

Instituto Agronômico do Paraná (1996). Amostragem de solo para análise química: plantio direto $e$ convencional, culturas perenes, várzeas, pastagens $e$ capineiras. Londrina: IAPAR. Circular, 90. Recuperado de http://www.iapar.br/arquivos/File/ zip pdf/CT90.pdf

Instituto Nacional de Meteorologia (2014). Banco de Dados Meteorológicos para Ensino e Pesquisa BDMEP. Brasília: INMET. Recuperado de http:// www.inmet.gov.br/portal/index.php?r=bdmep/ bdmep

Köppen, W., \& Geiger, R. (1928). Klimate der Erde. Gotha: Verlag Justus Perthes.
Lima, D. M., Jr., Rangel, A. H. N., Urbano, A. S., Oliveira, J. P. F., \& Maciel, M. V. (2014). Silagem de gramíneas tropicais não-graníferas. Agropecuária Científica no Semiárido, 10(2), 1-11. doi: 10.30969/ acsa.v10i2.284

Mabelebele, M., Siwela, M., Gous, R. M., \& Iji, P. A. (2015). Chemical composition and nutritive value of South African sorghum varieties as feed for broiler chickens. South African Journal Animal Science, 45(2), 206-213. doi: 10.4314/sajas.v45i2.12

Mahmood, A., \& Honermeier, B. (2012). Chemical composition and methane yield of sorghum cultivars with contrasting row spacing. Field Crops Research, 128(4), 27-33. doi: 10.1016/j.fcr.2011.12.010

McDonald, P., Edwards, R. A., Greenhalgh, J. F. D., Morgan, C. A., Sinclair, L. A., \& Wilkinson, R. G. (2011). Animal Nutrition (7nd ed.). Prentice Hall. Retrieved from http://gohardanehco.com/wpcontent/uploads/2014/02/Animal-Nutrition.pdf

Moraes, S. D., Jobim, C. C., Silva, M. S., Marquardt, F. I. (2013). Produção e composição química de híbridos de sorgo e de milho para silagem. Revista Brasileira de Saúde e Produção Animal, 14(4), 624-634. doi: 10.1590/S1519-99402013000400002

Neumann, M., Restle, J., Alves, D. C., Fo., Brondani, I. L., Pellegrini, L. G., \& Freitas, A. K. (2002). Avaliação do valor nutritivo da planta e da silagem de diferentes híbridos de sorgo (Sorghum bicolor, L. Moench). Revista Brasileira de Zootecnia, 31(1), 293-301. doi: 10.1590/S1516-35982002000200002

Neves, A. L. S., Santos, R. D., Pereira, L. G. R., Tabosa, J. N., Almeida, M. R. M., Rodrigues, J. A. S.,... Verneque, R. S. (2014). Agronomic characteristics of sorghum cultivars for silage production in the agreste of pernambuco state. Revista Brasileira de Milho e Sorgo, 13(3), 382-390. doi: 10.18512/19806477/rbms.v13n3p382-390

Pedreira, M. S., Reis, R. A., Berchielli, T. T., Moreira, A. L., \& Coan, R. M. (2003). Características agronômicas e composição química de oito híbridos de sorgo [Sorghum bicolor (L.) Moench]. Revista Brasileira de Zootecnia, 32(5), 1083-1092. doi: 10.1590/S1516-35982003000500008

Perazzo, A. F., Santos, E. M., Pinho, R. M. A., Campos, F. S., Ramos, J. P. F., Aquino, M. M.,... Bezerra, H. F. C. (2013). Características agronômicas e eficiência do uso da chuva em cultivares de sorgo no semiárido. Ciência Rural, 43(10), 1771-1776. doi: 10.1590/ S0103-84782013001000007 
Pereira, I. A., F ${ }^{\circ}$., \& Rodrigues, J. A. S. (2015). Sorgo: o produtor pergunta, EMBRAPA responde. Brasília: EMBRAPA Cerrado. Recuperado de https://www. infoteca.cnptia.embrapa.br/infoteca/bitstream/doc/ 1019313/1/Sorgo500perguntas500respostas.pdf

Possenti, R. A., Ferrari, E., Jr., Bueno, M. S., Bianchini, D., Leinz, F. F., \& Rodrigues, C. F. (2005). Parâmetros bromatológicos e fermentativos das silagens de milho e girassol. Ciência Rural, 35(5), 1185-1189. doi: 10.1590/S0103-84782005000500031

Rodrigues, O., Fo., França, A. F. A., Oliveira, R. P., Oliveira, E. R., Rosa, B., Soares, T. V., \& Mello, S. Q. S. (2006). Produção e composição bromatológica de quatro híbridos de sorgo forrageiro [Sorghum bicolor (L.) Moench] submetidos a três doses de nitrogênio. Ciência Animal Brasileira, 7(1), 37-48.

Silva, F. S. (2011). Manual de análises químicas de solos, plantas e fertilizantes (2nd ed.). Brasília, DF: EMBRAPA.

Silva, R., Santos, A., Tabosa, J. N., Gomes, F., \& Almeida, C. A. (2012). Avaliação de diferentes genótipos de sorgo para forragem e silagem. Revista Brasileira de Milho e Sorgo, 11(3), 225-233. doi: 10.18512/19806477/rbms.v11n3p225-233

Silva, T. C., Santos, E. M., Azevedo, J. A. G., Edvan, R. L., Perazzo, A. F., Pinho, R. M. A.,... Silva, D. S. (2011). Agronomic divergence of sorghum hybrids for silage yield in the semiarid region of Paraiba. Revista Brasileira de Zootecnia, 40(9), 1886-1893. doi: 10.1590/S1516-35982011000900007
Silva, T. C., Santos, E. M., Macedo, C. H. O., Lima, M. A., Bezerra, H. F. C., Azevêdo, J. A. G.,... Oliveira, J. S. (2012). Divergence of the fermentative and bromatological characteristics of 25 sorghum hybrid silages. Revista Brasileira de Zootecnia, 41(5), 11271133. doi: 10.1590/S1516-35982012000500007

Simili, F. F., Lima, M. L. P., Medeiros, M. I. M., Paz, C. C. P., \& Reis, R. A. (2014). Degradabilidade in situ do híbrido de sorgo e do capim-tanzania em vacas suplementadas no outono. Boletim de Indústria Animal, 71(2), 127-134. doi: 10.17523/bia. v71n2p127

Sousa, D. M. G., \& Lobato, E. (2004). Cerrado: correção do solo e adubação (2nd ed.). Brasília: EMBRAPA Cerrados.

Tazoe, Y., Sazuka, T., Yamaguchi, M., Saito, C., Ikeuchi, M., Kanno, K.,... Makino, A. (2016). Growth properties and biomass production in the hybrid $\mathrm{C}_{4}$ crop Sorghum bicolor. Plant Cell Physiology, 57(5), 944-952. doi: $10.1093 / \mathrm{pcp} / \mathrm{pcv} 158$

Van Soest, P. J. (1994). Nutritional ecology of the ruminant (2nd ed.). Ithaca: Cornell University Press.

Van Soest, P. J., Robertson, J. B., \& Lewis, B. A. (1991). Methods for dietary fiber, neutral detergent fiber, and nonstarch polysaccharides in relation to animal nutrition. Journal of Dairy Science, 74(10), 35833597. doi: 10.3168/jds.S0022-0302(91)78551-2

Xiao-Ping, L., Jin-Feng, Y., Cui-Ping, G., \& Acharya, S. (2011). Quantitative trait loci analysis of economically importante traits in Sorghum bicolor x S. sudanense hybrid. Canadian Journal of Plant Science, 91(1), 81-90. doi: 10.4141/cjps09112 